\title{
A Guide To Practical Implementation Of Wavelet Filters For Image De-Noising
}

\author{
Shanthi KJ DK Ravish \\ Dept. of Medical Electronics Dr. Ambedkar Institute of Technology Bangalore, India
}

\begin{abstract}
Restoration filters are used to recover an image that has been degraded by using a priori knowledge of the degradation phenomenon. This paper aims to provide a comparative study of both qualitative and quantitative performance of the restoration filters namely wavelet filter, median filter and wiener filter of which detailed study and analysis of the wavelet based noise removal is undertaken. The paper deals with the implementing the 2 level wavelet filters with all practical details. One of the main reasons for degradation of images is noise interference. The image is tested for speckle noise, Gaussian noise and salt and pepper noise. Standard test images in MATLAB are used and the filters are implemented using MATLAB functions with standard MATLAB images.
\end{abstract}

Keywords: image, Denoising, Wavelet filters, Haar,DWT, Decomposition

\section{Introduction}

The objective of restoration is to improve a given image in some predefined sense. Although there are areas of overlap between image enhancement and image restoration, the former is largely a subjective process while image restoration is for the most part an objective process. Restoration attempts to reconstruct or recover an image that has been degraded by using a priori knowledge of the degradation phenomenon. Thus restoration techniques are oriented toward modeling the degradation and applying the inverse process in order to recover the original image. The purpose of image restoration is to compensate for or undo defects which degrade an image. Degradation comes in many forms such as motion blur, noise, and camera misaligned focus. In cases like motion blur, it is possible to come up with a very good estimate of the actual blurring function and undo the blur to restore the original image [1]. In cases where the image is corrupted by noise, the best we may hope to do is to compensate for the degradation it caused.

Digital images play an important role both in day to-day applications, such as, satellite television, magnetic resonance imaging, computer tomography as well as in areas of research and technology such as geographical information systems and astronomy. Generally, data sets collected by image sensors are contaminated by noise. Imperfect instruments, problems with the data acquisition process, and interfering natural phenomena can all degrade the data of interest. Furthermore, noise can be introduced by transmission errors and compression. Thus, denoising is often a necessary and the first step to be taken before the image data is analyzed. It is necessary to apply an efficient restoration technique to get a model for such data corruption. Digital images are prone to a variety of types of noise. There are several ways that noise can be introduced into an image, depending on how the image is created. There are several types of noise models that degrade the image. Certain authors specify methods to restore the images without any noise model and called blind model [2]

The rest of the paper is organized such that section I.A briefs the noise model, section II describes the wavelet transform. Section III gives the methodology and the implementation of the algorithm. Section IV discusses the results of the Haar wavelet and subsequent threshlolding and inverse process.

\section{A. Noise models}

The objective of modeling is to characterize the structures and the patterns in a signal or a noise process. The simplest method for noise modeling, often used in current practice, is to estimate the noise statistics from the signal-inactive periods. In optimal Bayesian signal processing methods, a set of probability models are trained for the signal and the noise processes. The models are then used for the decoding of the underlying states of the signal and noise, and for noisy signal recognition and enhancement. Speckle noise effects are studied in this paper, it is a multiplicative noise and signal dependent noise. [3] Details the aspects of mathematical modeling of multiplicative speckle noise. Noise and distortion are the main limiting factors in communication and measurement systems. Therefore the modeling and removal of the effects of noise and distortion have been at the core of the theory and practice of communications and signal processing. Noise can be defined as an unwanted signal that interferes with the communication or measurement of another signal. A noise itself is a signal that conveys information regarding the source of the noise. There are different types of noises:- Thermal Noise, White Noise, Shot Noise, Colored Noise, Electromagnetic Noise, Impulsive Noise etc. The noise gets added to an image during transmission [4] and also at different stages of processing. 


\section{Wavelet Transform}

The wavelet filter discussed here is Haar wavelet. The two level discrete wavelet transform of the image is taken. Most of the noise will be represented by wavelet coefficients at the finer scales. We discard these coefficients below a threshold value and thus filtering is achieved. The Inverse DWT of the threshold coefficients is the denoised image. It has been found that such denoising is effective in that although noise is suppressed, edge features are retained without much damage. There are two types of thresholding hard and soft thresholding. In this paper hard thresholding is implemented. There are modifications in threshold include dual threshold [5] and adaptive threshold [6]

A wavelet is a waveform of limited duration that has an average value of zero. Wavelets are irregular, of limited duration, and often non symmetrical. They are better at describing anomalies, pulses and other events that start and stop within the signal. Some wavelets called crude wavelets are designed by a mathematical expression and are drawn as continuous and infinite. But to use them with digital signals they must first be converted to wavelet filters having finite number of discrete points [7]. Wavelets maintains spatial (temporal) as well as frequency information. Many classes of functions can be expanded in a relatively small number of wavelet basis functions while keeping most of their information content. These two properties make wavelets an excellent tool for noise filtering and data compression. Wavelet filters provide a tradeoff between Filters have to be selected based on the tradeoff between frequency and spatial domain [8]. Some of the main wavelets families are.

Haar: Any discussion of wavelets begins with Haar wavelet, the first and simplest. Haar wavelet is discontinuous, and resembles a step function. It uses a two bank filter system a low pass and high pass [9].

\section{Methodology}

The block diagram in fig. 1 gives the overview of the implementation of the methodology proposed in the system.

\section{B. Forward DWT level 1 decomposition}

Step 1: The noised image is used for the synthesis and it is passed as input.

Step 2: The input noised image is then passed through decomposition low pass and high pass filters using Haar wavelet. It uses a two bank filter system a low pass and high pass. The decomposition low pass and high pass filters using Haar wavelet can be given as:

i. Low pass decomposition ------ $0.707 *\left[\begin{array}{ll}1 & 1\end{array}\right]$

ii. High pass decomposition ------ $0.707 *\left[\begin{array}{ll}1 & -1\end{array}\right]$

Decomposition is done on each pixel taking row wise

Step 3: The decomposed images from the low pass and high pass filters are then down sampled by 2 column wise.

Step 4: The down sampling by 2 is done by taking only the alternate pixel values column wise. To be more detail taking only the even indexed columns and rejecting the odd ones.

Step 5: The each down sampled output from the low pass and high pass filters are then passed through decomposition low pass and high pass filter by taking each pixel value taking column wise. Occasionally researchers go for multi channel filters [10].

Step 6: Thus a total of 4 outputs can be obtained from both the input to the low pass and high pass decomposition filters column wise.

Step 7: These four outputs of the filters give the four decomposition coefficients. These are called level 1 decomposition coefficients

They are:

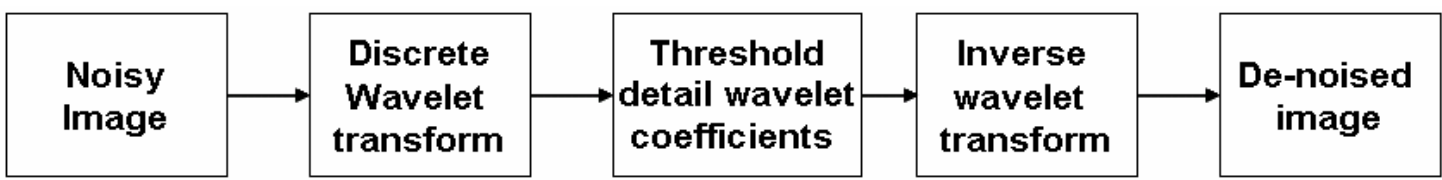

Figure 1. Block Diagram showing the Image denoising system

1. Approximation coefficient

2. Horizontal detail coefficient

3. Vertical detail coefficient

4. Diagonal detail coefficient

From the above said steps, the approximation coefficient is the low pass -low pass section, Horizontal is the low pass- high pass section, Vertical is high pass-low pass section and diagonal is the high pass- high pass section. Hence the last three coefficients consist of the noisy high pass components which are to be applied 
threshold. For much better decomposition we move to level 2 decomposition. In which the approximation coefficient in the first level is again decomposed in to further coefficients by repeating the same steps above.

\section{Forward DWT level 2 decomposition}

The Step 1: In level 2 decomposition, same as the level 1 decomposition, only difference is that the input applied is the level 1 approximation coefficient.

Step 2: The steps from 2 to 7 of the forward DWT level 1 algorithm is repeated.

Step 3: Performing the above steps will result in the decomposition of the level 2 decomposition coefficients.

\section{Applying Threshold}

This algorithm implements hard thesholding to the coefficients.

Step 1: Threshold is selected based on the analysis of the histogram of the decomposition coefficients. A threshold is determined in such a way that noise intensities are reduced.

Step 2: Hard thresholding is implemented for the decomposition coefficient. The equations of the hard thresholding is given as:

Hard thresholding sets any coefficient less than or equal to the threshold to zero.

(If (coeff.[i] > thresh) then coeff. [i] $=0$

Step 3: The Threshold is applied to the level 1 decomposition coefficients and level 2 decomposition coefficients. Step 4: Apply the Inverse discrete wavelet transform

\section{E. Inverse Discrete Wavelet Transform}

Step 1: With the threshold applied decomposition coefficients in the level 2 along with the approximation coefficient is up sampled column wise. Up sampling column wise is done inserting zeros at alternate pixels i.e. adding zeros at odd indexed columns

Step 2: The approximation coefficient and horizontal detail coefficient is passed through a set 1 column wise low pass and high pass reconstruction filter by taking pixels column wise

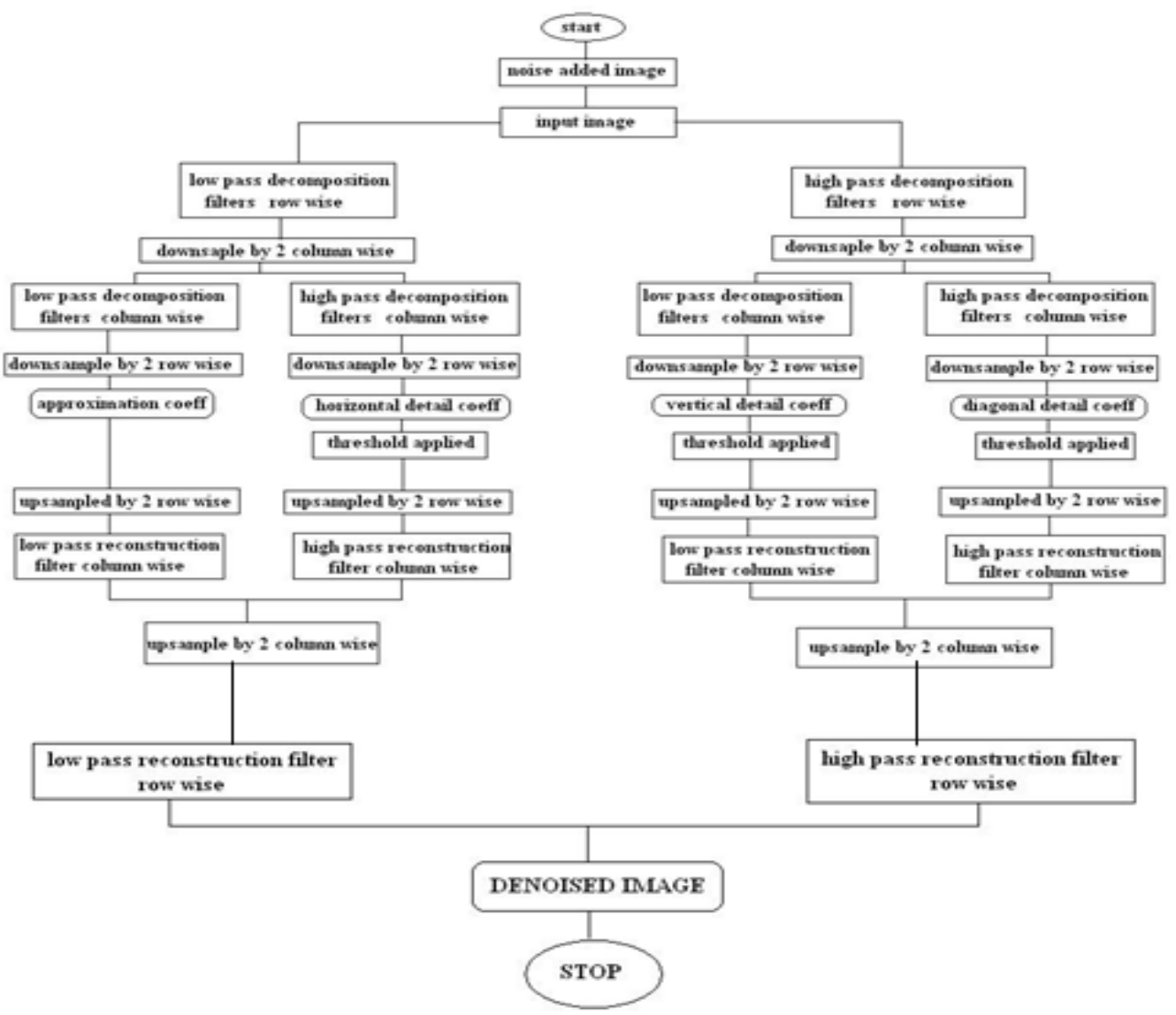

Figure 2. Flow Chart depicting the design process for noise removal 
The vertical and diagonal detail coefficients are passed through set 2 column wise low pass and high pass reconstruction filters.

Step 3: The output of the set 1 low pass and high pass filters are added together meanwhile output of the set 2 high pass and low pass are added together.

Step 4: Both the added images are then up sampled row wise. Up sampling in row wise is done by adding zeros at alternate pixels .i.e. adding zeros at the odd indexed rows.

Step 5: Both the up sampled outputs are then passed through a low pass and high pass reconstruction filters by taking pixels in row wise

Step 6: The output of the reconstruction filters are then added together to get the approximation coefficient of level 1.

Step 7: With this approximation coefficient at level 1 and the level 2 decomposition coefficients repeat the steps from step 1 through 6

Step 8: The output will give the de noised image.

\section{Result \& Discussion}

The algorithm was coded using MATLAB. Standard test images from MATLAB database were made use. Noisy images were generated by adding speckle noise with two different values of variance $0.2 \& 0.3$. The noises were removed by applying the hard threshold after performing two level decomposition. The program was also tested for salt \& pepper noise and Gaussian noise. The wavelet filters were found more effective in speckle noise. After performing two level decomposition. The program was also tested for salt \& pepper noise and Gaussian noise. The wavelet filters were found more effective in speckle noise Speckle noise is a multiplicative noise and it is signal dependent. The speckle noise unlike additive noise is a difficult one to remove. The other noise such as Gaussian and salt \& pepper noise, however wavelet filters were not proved to be effective. Where median filter and Wiener filters proved to be more effective. Hence wavelet filters present promise for filtering in real time applications of image processing and filtering for images such as MRI images.

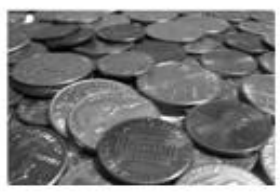

a) Original

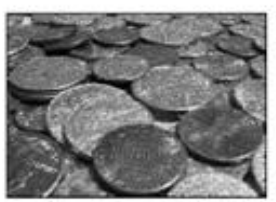

b) Noisy image: Speckle noise 0.2

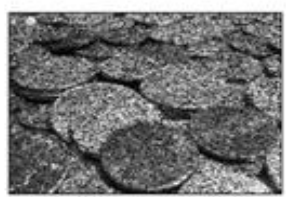

d) Noisy image: Speckle noise 0.3

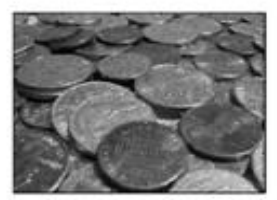

c) Filtered image

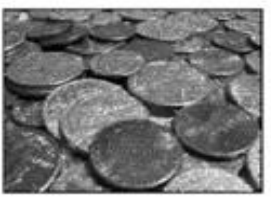

e) Filtered Image

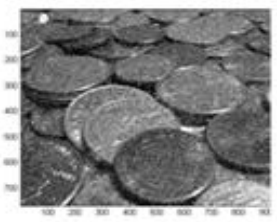

Salt \& Pepper noise

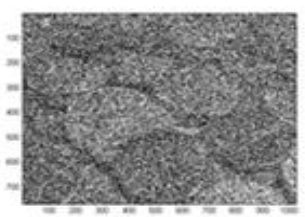

Gaussian Noise

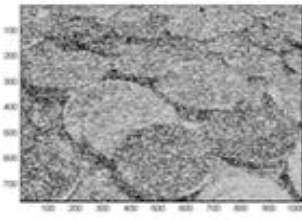

Wavelet Filtered

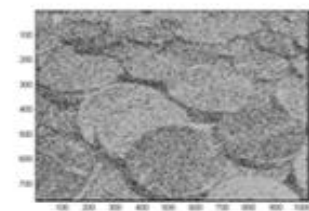

Wavelet Filtered

Figure 3. Noise degradation with various noise and wavelet filtered images

\section{References}

[1]. S. Motohashi, H. Senshiki, T. Goto and S. Hirano, Blind blur image restoration considering noise, 2016 IEEE 5th Global Conference on Consumer Electronics, Kyoto, 2016, pp. 1-2.doi: 10.1109/GCCE.2016.7800425

[2]. F. Zhu, G. Chen and P. A. Heng, From Noise Modeling to Blind Image Denoising, 2016 IEEE Conference on Computer Vision and Pattern Recognition (CVPR), Las Vegas, NV, 2016, pp. 420-429.doi: 10.1109/CVPR.2016.52

[3]. M. Hacini, K. Djemal and F. Hachouf, Speckled images restoration filter based on weighted multiplicative regularization approach $20123 r d$ International Conference on Image Processing Theory, Tools and Applications (IPTA), Istanbul, 2012, pp. 111-115. doi: 10.1109/IPTA.2012.6469506

[4]. M. Tayel, A. Gamal and H. Shawky, Denoising of Stego-images for different noise models, 2015 17th International Conference on Advanced Communication Technology (ICACT), Seoul, 2015, pp. 65-69. 
[5]. Mitiche, A. B. Houda Adamou-Mitiche and H. Naimi, Medical image denoising using dual tree complex thresholding wavelet transform, 2013 IEEE Jordan Conference on Applied Electrical Engineering and Computing Technologies (AEECT), Amman, 2013, pp. 1-5.doi: 10.1109/AEECT.2013.6716477

[6]. L. Dong, Adaptive image denoising using wavelet thresholding, 2013 IEEE Third International Conference on Information Science and Technology (ICIST), Yangzhou, 2013, pp. 854-857.doi: 10.1109/ICIST.2013.6747675

[7]. D Lee Fugal Conceptual Wavelets in Digital Signal Processing

[8]. F. Nicolier, G. Millon and E. Baudrier, A wavelet filter criterion for an a-priori evaluation of wavelet coding and denoising performances Signal Processing and Its Applications, 2003. Proceedings. Seventh International Symposium on, 2003, pp. 475-478 vol.2. doi: 10.1109/ISSPA.2003.1224917

[9]. Design of wavelet filters for DWMT systems Future Communication Networks (ICFCN), 2012 International Conference on April 2012 Baghdad pp 7 - 11 978-1-4673-0259-3

[10]. Lin Zhenxian, A new framework of M-channels lifting wavelet filter in image processing, Computer Science and Automation Engineering (CSAE), 2011 IEEE International Conference on, Shanghai, 2011, pp. 133-136. doi: 10.1109/CSAE.2011.5952650 